The role of self-disgust in eating psychopathology in overweight and obesity:

Can self-compassion be useful?

Lara Palmeira, José Pinto Gouveia, \&Marina Cunha 2017 Journal of Health Psychology Advance Online Publication doi: $10.1177 / 1359105317702212$ 


\title{
The role of self-disgust in eating psychopathology in overweight and obesity:
}

\section{Can self-compassion be useful?}

\author{
Lara Palmeira, José Pinto Gouveia, \& Marina Cunha
}

\begin{abstract}
This study explores the relationship between self-disgust and eating psychopathology and whether self-compassion plays a mediator role on this relationship. Participants were 203 adults, from both genders, with overweight and obesity $\left(\mathrm{M}_{\mathrm{BMI}}=31.17, \mathrm{SD}=5.43\right)$. Women reported higher levels of self-disgust and eating psychopathology and lower levels of self-compassion than men. Path analysis' results suggested that the effect of self-disgust on eating psychopathology occurred partially through one's inability to be self-compassionate. Results highlight the damaging role of self-disgust on eating psychopathology and the importance of developing a more compassionate attitude towards the self to promote healthy eating behaviors.
\end{abstract}

KEY-WORDS: Self-disgust; Self-compassion; Overweight and Obesity; Eating psychopathology; path analysis 


\section{INTRODUCTION}

Evolutionarily, disgust refers to feelings of revulsion (Rozin, Lowery, Imada, \& Haidt., 1999) and is an aversive response rooted in the threat-protection system that signals possible contamination or attacks, aiming to avoid diseases and protect us from ingesting harmful or poisoned substances (Gilbert, 2015; Oaten, Stevenson, \& Case, 2009). It motivates avoidancebased responses, such as moving away, getting rid of or eradicating what is dangerous or disgusting (Rozin et al., 1999). Nevertheless, disgust evolved to be a multifaceted and heterogeneous emotional construct shaped through sociocultural learning, that can be prompted by different stimuli (Power \& Dalgleish, 2008; Rozin et al., 1999), including: social deviance and immoral behavior, interpersonal relationships (being disgusted by other's behaviors or presentations) and self-judgements (regarding one's body, emotions, thoughts, behaviors or even the self) (Chapman \& Anderson, 2012; Ille, Schögglb, Kapfhammer, Arendasya, Sommera, \& Schienlea, 2014; Gilbert, 2015; Overton, Markland, Taggart, Bagshaw, \& Simpson, 2008) and lead to distinct responses. Although the disgust response serves an important and adaptive function in the external world, feelings of disgust may be generalized and directed towards the self when some aspects are seen as toxic, repugnant and dangerous (Gilbert, 2015; Overton et al., 2008; Power \& Dalgleish, 2008).

Self-disgust or self-loathing is considered to arise from the self-directed generalization of the basic disgust response and relates to feelings of aversion, deep grief or even repugnance towards the one's self, reflecting a noxious, harsh and embodied feeling state (Roberts \& Goldenberg, 2007). It is considered a stable and dysfunctional psychological phenomenon that encloses two interrelated domains of the self (physical and behavioral) and that is intrinsically dependent on one's social environment (Powell, Simpson, \& Overton, 2013). Even though self-disgust origins are yet to be fully understood, it is likely that it arises from social learning experiences such as: disgust-related criticism, internalization of other's disgust reactions and negative social comparisons, where the individuals learn what attributes others consider physically or socially repulsive (Powell, Simpson, \& Overton, 2015). Moreover, self-disgust is intrinsically linked to other threaten-based emotions, such as anger, fear, and shame that when directed towards one's self may be extremely pathogenic (Gilbert, 2015; Powell et al., 2015). It is unclear whether selfdisgust represents a severe form of shame or whether shame and self-disgust are variants of the disgust response (Roberts \& Goldenberg, 2007). Nevertheless, several authors have been claiming that self-disgust reflects an important and distinct construct that deserves more empirical attention (Roberts \& Goldenberg, 2007; Powell et al., 2015).

According to Gilbert (2005), individuals use identical psychological mechanisms to deal with both external and internal attacks and threats. Although disgust tends to elicit the urge to get rid of, avoid or reject what is considered disgusting, this can be particularly difficult when the 
stimuli that elicit the disgust feeling is a part (or the whole) self (Espeset, Gulliksen, Nordbø, Skårderud, \& Holte, 2012). Nonetheless, individuals can still engage in avoidance behaviors, such as avoiding touching or looking at themselves or being looked by others, masking the disgusting aspects of themselves or even trying to distract themselves from the object of disgust (Espeset et al., 2012; Powell et al., 2013). These avoidance-based strategies tend to yield paradoxical effects and contribute to increase or maintain the disgust response (Powell et al., 2015). Given that selfdisgust is particularly enduring and that avoidance-based strategies seem to produce unwanted effects, individuals with self-disgust may become somehow trapped with the desire to get rid of those parts considered disgusting (Powell et al., 2013).

Until now, research on self-disgust is still scant and its relationship with psychopathology needs to be further explored. Nonetheless, recently, in a longitudinal study, self-disgust has been found to be involved in the genesis of depressive experience, as an important predictor of depressive symptoms (Powell et al., 2013). Self-disgust has also been related to unhealthy eating behaviors (e.g. restrictive eating, purging, and vomiting) body dissatisfaction, urge to lose weight, being often directed at undesirable and unattractive body features (Espeset et al., 2012; Powell, Overton, \& Simpson, 2014; Shanmugarajah, Gaind, Clarke, \& Butler, 2012). Moreover, evidence suggests that individuals with eating disorders may use unhealthy eating behaviors to avoid or regulate negative affect and difficult emotions, such as shame, anger, sadness (Espeset et al., 2012; Fox, Grange \& Power, 2015; Power \& Dalgleish, 2008). In a study comparing patients with different psychological problems and healthy controls, Ille et al. (2014) found that those with psychological problems showed higher levels of self-disgust. Among those with mental problems, those with borderline personality and eating disorders presented the highest self-disgust levels. Furthermore, evidence suggests that self-disgust plays a crucial role in eating disorders maintenance, eliciting food, eating and body-related stimuli avoidance in women (Espeset et al., 2012; Fox \& Power, 2009). However, as far as we know the role of self-disgust in individuals with overweight and obesity has never been studied. Nevertheless, unveiling the role of selfdisgust in this population may be especially important given that, in modern society, physical appearance (particularly for women) has become a major source of social acceptance (Gilbert, Price, \& Allan, 1995) and is something that cannot be easily hidden from others. Thus, presenting a body that is different than the one that is socially valued and that others may view as disgusting can be threatening and become internalized, leading to feelings of shame and self-disgust. In turn, this increases one's vulnerability to engage in disordered eating patterns (Fox et al., 2015). Gilbert (2015) proposes that self-disgust encloses the desire to avoid the object of disgust, in order to become an acceptable and valued self within the social context.

On the other hand, research has been consistently highlighting the importance of developing self-compassion, i.e., having a warm, kind and accepting relationship with one's self as a protective emotional regulation process in mental health and well-being (MacBeth \& Gumley, 
2012; Neff, 2003). However, individuals that struggle with eating and weight problems seem to present difficulties in being self-compassionate towards themselves, especially when facing setbacks or failures (Adams \& Leary, 2007; Gilbert, Stubbs, Gale, Gilbert, Dunk, \& Thomson, 2014).

Evidence suggest that self-compassion may be linked to eating pathology in multiple ways (Braun, Park, \& Gorin, 2016). Self-compassion has been found to mediate the relationship between BMI, shame, body dissatisfaction and body image-related unfavorable social comparisons and eating psychopathological symptoms and quality-of-life in females from clinical and non-clinical samples (e.g., Duarte, Ferreira, Trindade, \& Pnto-Gouveia, 2015; Ferreira, PintoGouveia, \& Duarte, 2013). A German large study ( $\mathrm{N}=1158)$, with individuals with overweight and obesity, found that self-compassion mediated the relationship between weight self-stigma and global health (Hilbert, Braehler, Schmidt, Löwe, Häuser, \& Zenger, 2015). Taken together these studies emphasize self-compassion abilities as key resources for the adoption of healthy behaviours.

This study aims to explore the associations between self-disgust, self-compassion and eating psychopathological symptoms in individuals with overweight and obesity. We expect that selfdisgust would be positively related to eating psychopathology and negatively related to selfcompassion. Gender differences concerning all study's variables were also explored since literature has been highlighting that women are more prone to present eating psychopathology than men (e.g., Buchanan, Bluestein, Nappa, Woods, \& Depatie, 2013). Lastly, this study also investigated whether the ability to be self-compassionate mediated the relationship between selfdisgust feelings and eating psychopathological symptoms in individuals with overweight and obesity, while controlling gender and BMI.

\section{METHODS}

\section{Participants}

The sample comprised 203 individuals (50.2\% males and $49.8 \%$ females) with overweight and obesity $\left(M_{B M I}=31.17 ; S D_{B M I}=5.43\right)$ seeking nutritional treatment for weight loss in several public and private health care units in Portugal. Inclusion criteria were: being an adult (age > 18 years old) and having a BMI $\geq 25 \mathrm{Kg} / \mathrm{m} 2$. Participants presented a mean age of 40.08 ( $S D=$ $11.74)$, with a mean of $12.67(S D=3.74)$ years of education. No gender differences were found for age, $t_{(201)}=-1.228, p=.221$, years of education, $t_{(201)}=1.463, p=.145$ and BMI $t_{(201)}=-.451$, $p=.653$.

Concerning marital status $55.1 \%$ of the participants were married or living together, $36 \%$ were single, $8.4 \%$ were divorced and $0.5 \%$ were widowed. The majority $(68.5 \%)$ came from low to medium socio-economic status. 


\section{Procedures}

Before data collection ethical approval was obtained from all institutions that participated in the study. Participants were invited to the study by their own nutritionist on the day of their nutritional appointment. In the first page of the protocol, the study's goals, as well as the voluntary and confidential nature of the data, was stated. Participants were required to sign an informed consent before completing the self-reported questionnaires. The study's protocol took approximately 15 minutes to be completed.

\section{Measures}

Demographic Data. Participants reported their age, educational level, current height and weight. Participants were asked to report the weight of their current or previous nutritional appointment. Then BMI $\left(\mathrm{Wt} / \mathrm{Ht}^{2}\right)$ was calculated.

Multidimensional Self-Disgust Scale (MSDS; Carreiras, 2014) is a self-report measure with 33 items that assesses self-disgust concerning physical, behavioral and functioning aspects. It encloses four subscales: defensive activation (physiological component; "I have the feeling my body contracts"), cognitive-emotional (cognitive and emotional component; "I hate/despise that part of me"), avoidance (behavioral component; "I disguise/ dissimulate those aspects of me that I disgust") and exclusion (behaviors used to eliminate and exclude disgusting characteristics of the self; "I feel like cutting, burning, or excluding that part of myself"). Participants rate the frequency they experience each item on a 5 point Likert scale ( 0 - never and 4 - always). In the original study, conducted in a Portuguese sample, all subscales showed good internal consistency ( $\alpha=.95$ for defensive activation; $\alpha=.97$ for cognitive-emotional subscale; $\alpha=.77$ for exclusion and $\alpha=.84$ for avoidance), good convergent and divergent validities (Carreiras, 2014). For the present study, only the cognitive-emotional subscale was used to assess participant's disgust thoughts and feelings towards the self.

Self-Compassion Scale (SCS; Neff, 2003; Castilho, Pinto-Gouveia, \& Duarte, 2015) is a 26 items self-report measure that assesses the tendency to be compassionate toward the self when facing setbacks. The SCS encloses six subscales that measure three components of selfcompassion (self-kindness vs self-judgment, common humanity vs isolation and mindfulness vs over-identification) All items are rated on a 5 point Likert scale $(1=$ almost never; to $5=$ almost always). The total score reflects the mean of all items, with higher values indicating more selfcompassion. Both the original $(\alpha=.92)$ and the Portuguese version $(\alpha=.94)$ showed very good internal consistency (Neff, 2003; Castilho et al., 2015).

Eating Disorder Examination Questionnaire (EDE-Q; Fairburn \& Beglin, 1994; Machado, Martins, Vaz, Conceição, Pinto-Basto, \& Gonçalves, 2014) is a well-known 36-item self-report 
instrument that measures eating psychopathological symptoms using a 6 point rating scale. EDEQ has been consistently considered a reliable measure of eating psychopathology (Fairburn, 2008). The Portuguese version also presented very good internal consistency ( $\alpha=.94$; Machado et al., 2014). In this study, we only used the EDE-Q global score as we were interested in capturing eating psychopathological symptoms severity.

\section{Data analysis}

IBM SPSS Statistics 20 and AMOS software were used to perform all data analysis. Gender variable was dummy coded as $0=$ female and $1=$ male. Preliminary data analyses were executed to explore the adequacy of the data. Pearson correlation coefficients were calculated to explore the associations between BMI, self-disgust (cognitive-emotional subscale), self-compassion and eating psychopathological symptoms. Then independent $t$-tests were performed to explore gender differences in all study's variables (Field, 2013). Effect sizes were calculated with Cohen's d. Following Cohen's guidelines (1988 cited in Tabachnick \& Fidell, 2007) values $<.4=$ small effects; from .5 to .7 medium effects and $>.8$ large effect sizes.

Finally, to explore the mediator effect of self-compassion on the relationship between selfdisgust and eating psychopathological symptoms, while controlling for BMI and gender, a path analysis was used. Path analysis allows the simultaneous examination of structural relationships, as well as the examination of direct and indirect paths (e.g., Schumacker \& Lomax, 2004). Maximum Likelihood method was used since it allows for the estimation of all path coefficients and computes fit statistics. Model fit was assessed using several goodness-of-fit measures and recommended cut-points: Chi-Square $(\chi 2)$, Normed Chi-Square ( $\chi^{2} /$ d.f.), Comparative Fit Index (CFI $\geq .95$, desirable; Hu \& Bentler, 1998), Goodness of Fit Index (GFI $\geq .95$, desirable; Jöreskog \& Sörbom, 1996), Root Mean Square Error of Approximation (RMSEA $\leq .08$, acceptable fit; Kline, 2005) with a $95 \%$ confidence interval. The mediation effect was examined using bootstrap (2000 resamples) with $95 \%$ bias-corrected confidence interval. The effect is statistically significant at $\mathrm{p}<.05$ if zero is not included in the interval between the lower and the upper bound (Kline, 2005).

\section{RESULTS}

\section{Preliminary Data Analyses}

All variables showed acceptable skewness and kurtosis values below the recommend cutpoints (SK $<|3|$ and $\mathrm{Ku}<|8-10|)$. Also, multicollinearity was not identified as all variables had VIF $<5$. Finally, Mahalanobis distance statistic $\left(D^{2}\right)$ did not detect the presence of any outliers (Kline, 2005). 


\section{Correlation analysis}

Pearson's correlation analyses are displayed in Table 1. Results reveal that gender and BMI were not significantly correlated. Gender also showed negative and low associations with selfdisgust and EDE-Q and a low and positive correlation with self-compassion. BMI showed positive and low associations with self-disgust and EDE-Q. BMI was not significantly related to self-compassion. Self-disgust was negatively and moderately related to self-compassion and positively and moderately associated with EDE-Q. Lastly, self-compassion was negatively and moderately correlated with EDE-Q.

\section{Table 1}

Means (M), standard deviations (SD),, Alpha coefficients and Pearson moment correlation for all study's variables $(N=203)$

\begin{tabular}{lccccccc}
\hline \multicolumn{1}{c}{ Measures } & $\mathrm{M}$ & $\mathrm{SD}$ & $\alpha$ & 1 & 2 & 3 & 4 \\
\hline 1. Gender & - & - & - & - & & & \\
2. BMI & 31.17 & 5.43 & - & .03 & - & & \\
3. Self-disgust & 8.04 & 7.44 & .94 &.$-18^{* *}$ & $.27 * * *$ & - & \\
4. Self-compassion & 3.25 & 0.60 & .92 &.$-28^{* * *}$ & -.11 & $-.59 * * *$ & - \\
5. EDE-Q & 1.77 & 1.25 & .94 & $.21 * *$ & $.37 * * *$ & $.65 * * *$ & $-.48^{* * *}$ \\
\hline Note $* * p<01 * * * p<001 ;$ BMI $=$ Body Mass Index; EDE-Q = Eating Disorder Examination Questionnaire.
\end{tabular}

\section{Gender differences}

To explore differences regarding BMI, self-disgust, self-compassion and eating psychopathological symptoms between females and males, independent samples $t$-test were performed. Means, standard deviations, $t$-test differences and Cohen's $\mathrm{d}$ for all variables for each gender are displayed in Table 2. As can be seen, significant differences were found for all study's variables, except for BMI $(t(201)=-0.451, p=.653 ; d=.06)$. Overall, females reported higher levels of self-disgust $(t(201)=2.625, p=.009 ; d=.37)$ and eating psychopathological symptoms $(t(201)=4.116, p \leq .001 ; d=.58)$. On the contrary, men presented higher levels of selfcompassion abilities $(t(201)=-3.076, p=.002 ; d=.43)$. All differences represent small effect sizes, with the exception for eating psychopathological symptoms, where the effect size was medium. 
Table 2

Means (M), standard deviations (SD), t-test differences by gender for all variables and Cohen's $d$ effect size $(N=203)$

\begin{tabular}{lcccccccc}
\hline & $\begin{array}{c}\text { Female } \\
(\boldsymbol{n}=\mathbf{1 0 1})\end{array}$ & \multicolumn{2}{c}{$\begin{array}{c}\text { Male } \\
(\boldsymbol{n}=\mathbf{1 0 2})\end{array}$} & & & \\
& $\boldsymbol{M}$ & $\boldsymbol{S D}$ & $\boldsymbol{M}$ & $\boldsymbol{S D}$ & $\boldsymbol{t}(\boldsymbol{d} \boldsymbol{d})$ & $\boldsymbol{p}$ & Cohen's d \\
& 31.00 & 5.42 & 31.34 & 5.46 & $-.451(201)$ & .653 & .06 \\
\hline BMI & 9.40 & 8.40 & 6.70 & 6.56 & $2.625(201)$ & .009 & .37 \\
Self-disgust & 3.12 & 0.61 & 3.38 & 0.56 & $-3.076(201)$ & .002 & .43 \\
Self-compassion & & & & & & & \\
EDE-Q & 2.12 & 1.23 & 1.42 & 1.19 & $4.116(201)$ & $<.001$ & .58 \\
\hline
\end{tabular}

Note . BMI = Body Mass Index EDE-Q = Eating Disorder Examination Questionnaire.

\section{Path analysis}

Path analysis was conducted in order to test the mediational role of self-compassion on the relationship between self-disgust and eating psychopathological symptoms, while controlling for the effect of BMI and gender. The model tested contained 15 parameters. As fully saturated models always have a perfect model fit, model fit indices were neither examined nor reported. All path coefficients were statistically significant with the exception of the direct path from BMI $\rightarrow$ self-compassion $(\mathrm{b}=0.005 ; \mathrm{SE}=0.006 ; \mathrm{Z}=0.718 ; \mathrm{p}=.473)$ and the direct path from gender $\rightarrow$ self-compassion $(b=0.129 ; \mathrm{SE}=0.068 ; \mathrm{Z}=1.888 ; \mathrm{p}=.059)$ that were non-significant and were progressively removed. The final model (Figure 1) presented a very good model fit: $\chi^{2}(2)=4.049$, $p=.473 ; \chi^{2} /$ d.f. $=2.025 ; \mathrm{CFI}=.992 ; \mathrm{GFI}=.992 ; \mathrm{RMSEA}=.071,[\mathrm{CI}=.000 ; .172] ; p=.266$. 


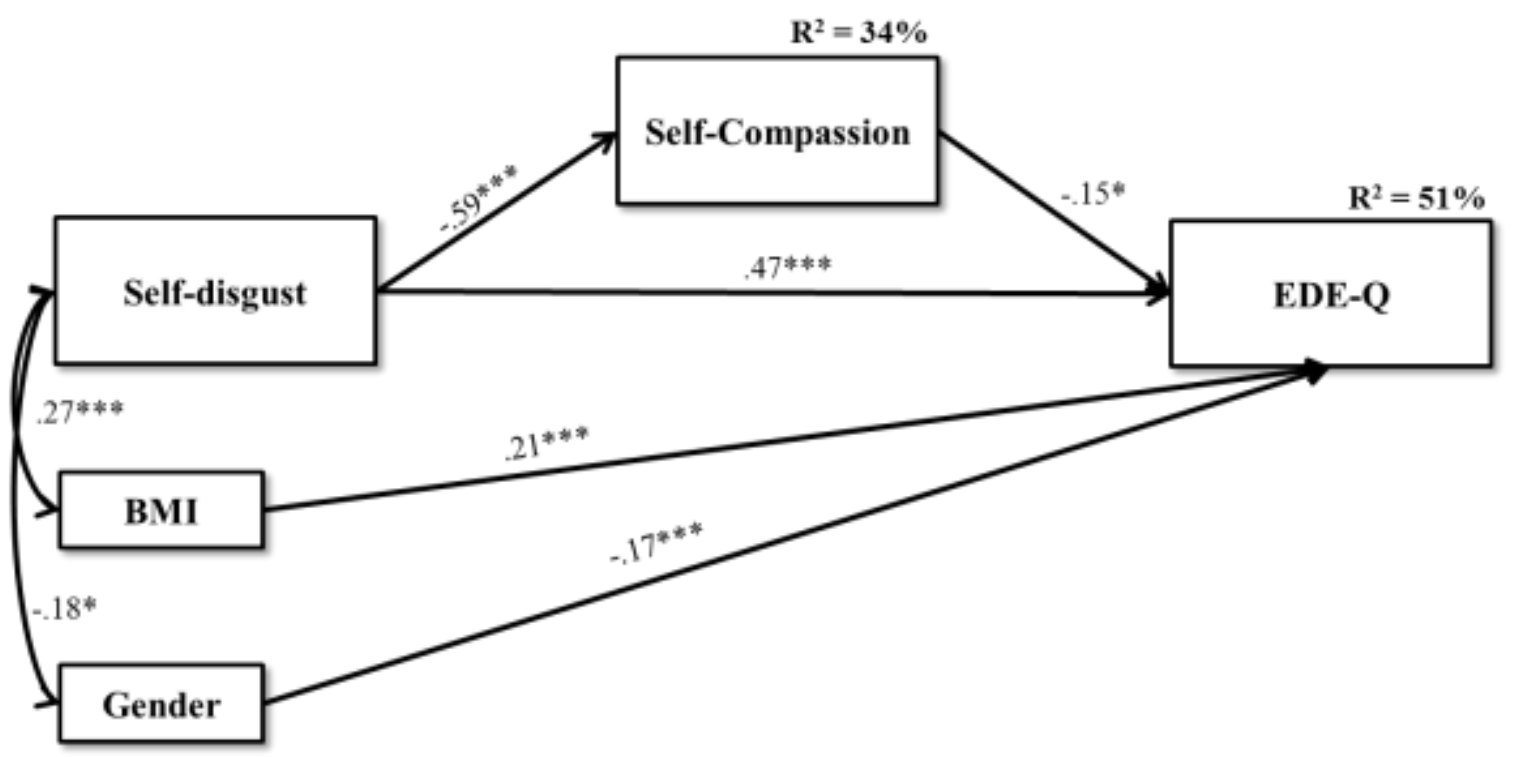

Fig. 1. Graphic representation of the Mediation Model $(N=203)$.

Note. ${ }^{* *} p \leq .01 ;{ }^{* * *} p \leq .001 ;$ Standardized path coefficients are presented.

Self-disgust was significantly correlated with both BMI $(\mathrm{r}=.27, p \leq .001)$ and gender $(\mathrm{r}=$ $.18, p=-011)$. Concerning the meditation analysis, results showed that self-disgust had a significant direct ( $\beta=0.465$ based on $95 \%$ CI: $0.326 ; 0.590, p=.001)$ and an indirect effect on EDE-Q through self-compassion ( $\beta=0.085$ based on $95 \%$ CI: $0.007 ; 0.159, p=.033)$. The total effect (that represent the sum of the standardized direct and indirect effects) of self-disgust on EDE-Q was $\beta=0.551$ based on $95 \% \mathrm{CI}(0.438 ; 0.640, p=.001)$. Moreover, there was a significant direct effect of self-disgust on self-compassion $(\beta=-0.586$, based on $95 \%$ CI: $-0.666 ;-0.498, p$ $=.001) ; 2)$ and a significant direct effect of self-compassion on EDE-Q $(\beta=-0.146$, based on $95 \%$ CI: $-0.272 ;-0.012, p=.033)$; Also, BMI ( $\beta=0.237$, based on 95\% CI: $0.113 ; 0.338, p=.001)$ and gender ( $\beta=-0.172$, based on $95 \% \mathrm{CI}$ : $-0.267 ;-0.068, p=.001)$ both presented significant direct effects on EDE-Q. The final model accounted for $34 \%$ of self-compassion and $51 \%$ of EDE-Q.

\section{DISCUSSION}

Self-disgust has been described as an enduring feeling of aversion, deep grief or repugnance towards some parts of the self (e.g. physical, psychological or behavioral), that stimulates avoidance-based responses (Roberts \& Goldenberg, 2007). Especially when directed at relatively stable attributes of the self (e.g. body weight), self-disgust seem to be particularly maladaptive and associated with disordered eating (Espeset et al., 2012; Fox \& Power, 2009). However, research on self-disgust is still in its early stages. As far as we know this is the first study that 
explored self-disgust in a sample of individuals with overweight and obesity seeking treatment for weight loss.

Results revealed that self-disgust was positively and moderately associated with eating psychopathological symptoms, whereas BMI only revealed positive but low associations with self-disgust and eating psychopathological symptoms. This result mirrors the results found in previous studies with non-clinical and eating disorders samples that showed that self-disgust was related to restrictive eating, purging, body dissatisfaction and urge to lose weight (Espeset et al., 2012; Powell et al., 2013). Contrarily, and as expected, the ability to have a warm and caring relationship with oneself was negatively associated with self-disgust and eating psychopathological symptoms and was not significantly related to BMI.

Consistent with previous findings, women presented higher levels of self-disgust and eating psychopathological symptoms and fewer self-compassion abilities than men. All gender differences reflected small effects, with the exception of the medium effect size found for eating psychopathological symptoms. In fact, literature points out suggest that women are more selfcritic and have fewer self-compassion skills than men (Neff, 2003; Yarnell, Stafford, Neff, Reilly, Knox, \& Mullarkey, 2015) and are more vulnerable to eating psychopathology (Buchanan et al., 2013). Likewise, although less studied, women present higher levels of self-disgust than men (e.g, Ille et al., 2014). These findings suggest that women are more prone to develop a negative and harmful internal relationship and may require differentiated interventions.

In addition, the present study highlights the role of self-disgust on eating psychopathological symptoms in people living with overweight and obesity. It seems that individuals that experience more self-disgust-related thoughts and emotions present more eating psychopathological symptoms. This is in line with the existent studies with patients with Anorexia and Bulimia Nervosa that suggest that self-disgust is closely linked with body-image dissatisfaction, avoidance, tendency to restrict food intake and purging behaviors (Espeset et al., 2012; Powell et al., 2014). Furthermore, these findings give empirical support to Gilbert's (2015) idea that trying to avoid or get rid of what is considered disgusting enfolds the wish to become accepted and valued in the eyes of others. Given the fact that one's weight and physical appearance is easily judged by others and that, especially for women, physical appearance is a crucial element for social acceptance, being considered overweight may present a threat to one's social acceptance and status (Gilbert et al., 1995; Simpson, Hillman, Crawford, \& Overton, 2010). In fact, Gilbert (2015) argued that if the self, or parts of the self, are seen as disgusting by others (e.g., weight or physical appearance), this view can become built in the self-system. Thus, self-disgust feelings may lead people to increase their weight control strategies and become more preoccupied with their weight, eating and body image as a way to be accepted and valued in their social context.

Consistent with previous research (Buchanan et al., 2013; Ferreira, Palmeira, \& Trindade, 2014), having a higher BMI and being female (although weakly) were directly related to higher 
levels of eating psychopathological symptoms. The fact that in our male sample the EDE-Q mean score was below the cut-off for eating psychopathology, may also account for the results found.

Moreover, in our model, the relationship between self-disgust and eating psychopathological symptoms was partially mediated by individual's inability to adopt a compassionate attitude towards themselves. Overall, the model tested accounted for $34 \%$ of self-compassion and $51 \%$ of eating psychopathological symptoms. This result points out that the effect of feeling disgust towards the self on disordered eating symptomatology seems to occur partially through the difficulty in accepting and having a warm and kind attitude towards oneself and one's imperfections. This is noteworthy as once acquired, self-disgust is considered hard to unlearn and that avoidance-based strategies tend to be ineffective (Powell, et al., 2015).

To sum up, this study highlights the harmful role of self-disgust on eating psychopathological symptoms in people living with overweight and obesity. It also points out that being selfcompassionate when facing failures or errors can be a useful resource in self-disgust and eating psychopathology. Still, research on self-disgust is recent and more studies are needed to better understand its origins and impact on the lives of people living with overweight and obesity. Future studies could continue to explore the impact of self-disgust particularly on binge eating and quality-of-life.

The present study encloses some limitations. This is a cross-sectional study, which precludes conclusions regarding causality. Clearly, longitudinal studies are needed to determine the directionality of the associations found. Likewise, we relied on participants self-reports (including height and weight), which may be biased and influenced the results. Additionally, we were unable to assess participant's medical and psychiatric history or the existence of eating disorders. Future studies should also take these aspects into account as they may influence the results found. Finally, the model tested is limited as it is likely that other variables (e.g., body dissatisfaction, selfcriticism) and other emotional regulation processes (e.g., decentering, experiential avoidance, cognitive fusion) may be involved in the relationship between self-disgust and eating psychopathological symptoms. However, we intentionally restrained this model to specifically explore the role of self-disgust and self-compassion abilities.

In conclusion, this study offers new insights for future research on self-disgust and stresses the importance of assessing and targeting self-disgust - an often neglected emotion - in adults (especially women) with overweight and obesity seeking treatment. Furthermore, our findings suggest the importance of fostering self-compassion skills, so individuals may develop a more detached and accepting relationship with their internal experiences, instead of being judgmental and becoming overidentified with them. In turn, this may help to decrease their eating psychopathological symptoms. 


\section{Funding}

This research was supported by the first author's Ph.D. Grant (SFRH/BD/84452/2012), sponsored by FCT (Portuguese Foundation for Science and Technology).

\section{Acknowledgments}

The authors would like to thank to the nutritionists that collaborated with the data collection and to all participants that took part in the study.

\section{Declaration of Conflicting Interests}

The authors declare that they have no conflicts of interest. 


\section{REFERENCES}

Adams, C. E. \& Leary, M. R. (2007). Promoting self-compassionate attitudes toward eating among restrictive and guilty eaters. Journal of Social and Clinical Psychology 26: 11201144. doi: 10.1521/jscp.2007.26.10.1120

Braun, T. D., Park, C. L., \& Gorin, A. (2016). Self-compassion, body image, and disordered eating: A review of the literature. Body Image, 17: 117-131. doi: http://dx.doi.org/10.1016/j.bodyim.2016.03.003

Buchanan, N. T., Bluestein, B. M., Nappa, A. C., Woods, K. C., \& Depatie, M. M. (2013). Exploring gender differences in body image, eating pathology, and sexual harassment. Body Image 10: 352- 360

Carreiras, D. (2014). The Toxicity of the Self: Developing a New Measure and Testing a Comprehensive Model of the Nature of Self-Disgust. Unpublished Master Dissertation. University of Coimbra. Available at: https://estudogeral.sib.uc.pt/handle/10316/27653

Castilho, P., Pinto-Gouveia, J., \& Duarte, J. (2015). Evaluating the Multifactor Structure of the Long and Short Versions of the Self-Compassion Scale in a Clinical Sample. Journal of Clinical Psychology, 71(9): 856-70. doi: 10.1002/jclp.22187.

Chapman, H. A., \& Anderson, A. K. (2012). Understanding disgust. Annals of the New York Academy of Sciences, 1251: 62-76.

Duarte, C., Ferreira, C., Trindade, I. A., \& Pinto-Gouveia, J. (2015). Body image and college women's quality-of-life: The importance of being self-compassion. Journal of Health Psychology, 20(6): 754-764. doi: 10.1177/1359105315573438.

Espeset, E. M. S., Gulliksen, K. S., Nordbø, R. H. S., Skårderud, F., \& Holte, A. (2012). The link between negative emotions and eating disorder behaviour in patients with anorexia nervosa. European Eating Disorders Review 20: 451-460. doi: 10.1002/erv.2183.

Fairburn, C. G. (2008). Cognitive behavior therapy and eating disorders. Guilford Press.

Fairburn, C. \& Beglin, S. (1994). Assessment of eating disorders: Interview or self-report questionnaire? International Journal of Eating Disorders 16(4): 363-370. doi:10.1002/1098-108X(199412)16:4<363::AID-EAT2260160405>3.0.CO;2-\#

Ferreira, C., Palmeira, L., \& Trindade, I. A. (2014). Turning eating psychopathology risk factors into action: The pervasive effect of body image-related cognitive fusion, Appetite, 80(1): 137-142. doi: 10.1016/j.appet.2014.05.019.

Ferreira, C., Pinto-Gouveia, J., \& Duarte, C. (2013). Self-compassion in the face of shame and body image dissatisfaction: Implications for eating disorders. Eating Behaviors 14: 207210. doi:10.1016/j.eatbeh.2013.01.005.

Field, A. (2013). Discovering Statistics using IBM SPSS Statistics. (4 ${ }^{\text {th }}$ ed.). Los Angeles, London: SAGE. 
Fox, J. R. \& Power, M. J. (2009). Eating disorders and multi-level models of emotion: An integrated model. Clinical Psychology \& Psychotherapy 16: 240-267.

Fox, J. R., Grange, N., \& Power, M. J. (2015). Self-disgust in eating disorders: A review of the literature and clinical implications. The revolting self: Perspectives on the psychological, social, and clinical implications of self-directed disgust, 167-186.

Gilbert, J., Stubbs, J., Gale, C., Gilbert, P., Dunk, L., \& Thomson, L. (2014). A qualitative study of the understanding and use of 'compassion focused coping strategies' in people who suffer from serious weight difficulties. Journal of Compassionate Health Care 1(9). doi: 10.1186/s40639-014-0009-5.

Gilbert, P. (2005). Compassion: Conceptualizations, research and use in psychotherapy. Routledge.

Gilbert, P. (2015). Self-Disgust, Self-Hatred and Compassion Focused Therapy. In Powell, P. A., Overton, P.G., \& Simpson, J. (eds) The revolting self: Perspectives on the Psychological, Social, and Clinical Implications of Self-Directed Disgust. Karnac Books, London.

Gilbert, P., Price, J., \& Allan, S. (1995). Social comparison, social attractiveness and evolution: How might they be related? New Ideas in Psychology 13: 149-165. http://dx.doi.org/10.1016/0732-118X(95)00002-X.

Hilbert, A., Braehler, E., Schmidt, R., Löwe, B., Häuser, W., \& Zenger, M. (2015). SelfCompassion as a Resource in the Self-Stigma Process of Overweight and Obese Individuals. Obesity Facts 8: 293-301. doi: 10.1159/000438681.

Hu, L. \& Bentler, P. M. (1998). Fit indices in covariance structure modeling: Sensitivity to underparameterized model misspecification. Psychological Methods, 3: 424-453.

Ille, R., Schögglb, H., Kapfhammer, H., Arendasya, M., Sommera, M., \& Schienlea, A. (2014). Self-disgust in mental disorders - symptom-related or disorder-specific? Comprehensive Psychiatry 55: 938-943. doi: 10.1016/j.comppsych.2013.12.020.

Jöreskog. K. G. \& Sörbom, D. (1996). LISREL 8 user's reference guide. Chicago: Scientific Software International.

Kline, R. B. (2005). Principles and Practice of Structural Equation Modeling (2nd ed.). 366, New York: Guilford.

MacBeth, A., \& Gumley, A. (2012). Exploring compassion: a meta-analysis of the association between self-compassion and psychopathology. Clinical Psychology Review 32 (6): 54552. doi: 10.1016/j.cpr.2012.06.003.

Machado, P., Martins, C., Vaz, A., Conceição, E., Pinto-Basto, A., \& Gonçalves, S. (2014). Eating Disorder Examination Questionnaire: Psychometric Properties and Norms for the Portuguese Population. European Eating Disorders Review 22 (6): 448-453. doi:10.1002/erv.2318. 
Neff, K. (2003). The development and validation of a scale to measure self-compassion. Self and Identity 2(3): 223-250. doi: 10.10080/15298860309027.

Oaten, M., Stevenson, R. J., \& Case, T. I. (2009). Disgust as a disease-avoidance mechanism. Psychological Bulletin 135: 303-321. doi: 10.1037/a0014823.

Overton, P. G., Markland, F.E., Taggart. H. S., Bagshaw, G. L., \& Simpson, J. (2008). Selfdisgust mediates the relationship between dysfunctional cognitions and depressive symptomatology. Emotion 8(3): 379-385. doi:10.1037/1528-3542.8.3.379.

Powell, P. A., Overton, P. G., \& Simpson, J. (2014). The Revolting Self: An Interpretative Phenomenological Analysis of the Experience of Self-Disgust in Females with Depressive Symptoms. Journal of Clinical Psychology 70(6): 562-578. doi: 10.1002/jclp.22049.

Powell, P. A., Simpson, J., \& Overton, P. G. (2013). When disgust leads to dysphoria: A threewave longitudinal study assessing the temporal relationship between self-disgust and depressive symptoms. Cognition \& Emotion 27(5): 900-913. doi: 10.1080/02699931.2013.767223.

Powell, P. A., Simpson, J., \& Overton, P. G. (2015). An introduction to the revolting self: Selfdisgust as an emotion schema. In Powell, P. A., Overton, P. G., \& Simpson, J. (eds). The revolting self: Perspectives on the Psychological, Social, and Clinical Implications of SelfDirected Disgust. Karnac Books, London.

Power, M. J. \& Dalgleish, T. (2008). Cognition and emotion: From order to disorder (2nd edition). Hove, UK: Psychology Press.

Rozin, P., Lowery, L., Imada, S., \& Haidt, J. (1999). The CAD triad hypothesis: A mapping between three moral emotions (contempt, anger, disgust) and three moral codes (community, autonomy, divinity). Journal of Personality and Social Psychology 76: 574586.

Roberts, T. A. \& Goldenberg, J. L. (2007). Wrestling with nature: An existential perspective on the body and gender in self-conscious emotions. In Tracy, J. L., Robins, R. W., \& Tangney, J. P. (eds.) The self-conscious emotions: theory and research. New York: Guildford, pp. 389-406.

Shanmugarajah, K., Gaind, S., Clarke, A., \& Butler, P. E. M. (2012). The role of disgust emotions in the observer response to facial disfigurement. Body Image 9: 455-461.

Simpson, J., Hillman, R., Crawford, T., \& Overton, P. G. (2010). Self-esteem and self-disgust both mediate the relationship between dysfunctional cognitions and depressive symptoms. Motivation and Emotion 34: 399-406.

Schumacker, R. E. \& Lomax, R. G. (2004). A beginner's guide to structural equation modeling (2nd edition). Mahwah, NJ: Lawrence Erlbaum Associates.

Tabachnick, B. \& Fidell, L. (2007). Using multivariate statistics. New York: Pearson Education Inc. 
Yarnell, L. M., Stafford, R. E., Neff, K. D., Reilly, E. D., Knox, M. C., \& Mullarkey, M. (2015). Meta-Analysis of Gender Differences in Self-Compassion. Self and Identity 14(5): 499520. doi: 10.1080/15298868.2015.10299 